\title{
(Q) Effect of postoperative continuation of antibiotic prophylaxis on the incidence of surgical site infection: a systematic review and meta-analysis
}

\author{
Stijn W de Jonge*, Quirine JJ Boldingh*, Joseph S Solomkin, E Patchen Dellinger, Matthias Egger, Georgia Salanti, Benedetta Allegranzi, \\ Marja A Boermeester
}

Lancet Infect Dis 2020; 20: 1182-92

Published Online May 26, 2020 https://doi.org/10.1016/ S1473-3099(20)30084-0

See Comment page 1110

*Contributed equally

Department of Surgery, Academic Medical Center, University of Amsterdam, Amsterdam, Netherlands ( $S$ W de Jonge MD, QJJ Boldingh MD,

Prof M A Boermeester MD); Department of Surgery, University of Cincinnati College of Medicine, Cincinnati, $\mathrm{OH}$ USA (Prof J S Solomkin MD);

Department of Surgery, University of Washington Medical Center, Seattle, WA, USA (Prof E P Dellinger); Institute for Social and

Preventive Medicine, University of Bern, Bern, Switzerland (Prof M Egger MD, Prof G Salanti PhD); and WHO Infection Prevention and Control Global Unit, Service Delivery and Safety, Geneva, Switzerland (B Allegranzi MD)

Correspondence to: Prof Marja A Boermeester, Academic Medical Center,

University of Amsterdam,

Amsterdam 1100 DD, Netherlands m.a.boermeester@amc.uva.nl

\section{Summary}

Background Antibiotic prophylaxis is frequently continued for 1 day or more after surgery to prevent surgical site infection. Continuing antibiotic prophylaxis after an operation might have no advantage compared with its immediate discontinuation, and it unnecessarily exposes patients to risks associated with antibiotic use. In 2016, WHO recommended discontinuation of antibiotic prophylaxis after surgery. We aimed to update the evidence that formed the basis for that recommendation.

Methods For this systematic review and meta-analysis, we searched MEDLINE, Embase, CINAHL, CENTRAL, and WHO regional medical databases for randomised controlled trials (RCTs) on postoperative antibiotic prophylaxis that were published from Jan 1, 1990, to July 24, 2018. RCTs comparing the effect of postoperative continuation versus discontinuation of antibiotic prophylaxis on the incidence of surgical site infection in patients undergoing any surgical procedure with an indication for antibiotic prophylaxis were eligible. The primary outcome was the effect of postoperative surgical antibiotic prophylaxis continuation versus its immediate discontinuation on the occurrence of surgical site infection, with a prespecified subgroup analysis for studies that did and did not adhere to current best practice standards for surgical antibiotic prophylaxis. We calculated summary relative risks (RRs) with corresponding 95\% CIs using a random effects model (DerSimonian and Laird). We evaluated heterogeneity with the $\chi^{2}$ test, $I^{2}$, and $\tau^{2}$, and visually assesed publication bias with a contour-enhanced funnel plot. This study is registered with PROSPERO, CRD42017060829.

Findings We identified 83 relevant RCTs, of which 52 RCTs with 19273 participants were included in the primary meta-analysis. The pooled RR of surgical site infection with postoperative continuation of antibiotic prophylaxis versus its immediate discontinuation was 0.89 (95\% CI $0.79-1.00)$, with low heterogeneity in effect size between studies $\left(\tau^{2}=0 \cdot 001, \chi^{2} \mathrm{p}=0 \cdot 46, I^{2}=0 \cdot 7 \%\right)$. Our prespecified subgroup analysis showed a significant association between the effect estimate and adherence to best practice standards of surgical antibiotic prophylaxis: the RR of surgical site infection was reduced with continued antibiotic prophylaxis after surgery compared with its immediate discontinuation in trials that did not meet best practice standards $(0 \cdot 79$ [95\% CI $0 \cdot 67-0.94])$ but not in trials that did $(1 \cdot 04$ [0 $85-1 \cdot 27]$ $\mathrm{p}=0.048)$. Whether studies adhered to best practice standards explained all variance in the pooled estimate from the primary meta-analysis.

Interpretation Overall, we identified no conclusive evidence for a benefit of postoperative continuation of antibiotic prophylaxis over its discontinuation. When best practice standards were followed, postoperative continuation of antibiotic prophylaxis did not yield any additional benefit in reducing the incidence of surgical site infection. These findings support WHO recommendations against this practice.

Funding None.

Copyright (C) 2020 World Health Organization. Published by Elsevier Ltd. All rights reserved.

\section{Introduction}

Antibiotic use is currently under scrutiny because of concerns about the emergence of antimicrobial resistance and other hazardous side-effects. ${ }^{1,2}$ Worldwide, approximately one in six in-hospital prescriptions for antibiotics is for surgical antibiotic prophylaxis, which is frequently continued for several days after surgery.,4 Although the effectiveness of appropriate antibiotic prophylaxis to prevent surgical site infections in indicated procedures is well established, ${ }^{5}$ increasingly evidence suggests that a single preoperative dose of antibiotics, with administration repeated intraoperatively when indicated, might be as effective as a prolonged postoperative regimen for a range of procedures..$^{6,7}$

Increased exposure to antibiotics has been associated with an increased risk of antimicrobial resistance, infection with Clostridioides difficile, and acute kidney injury, ${ }^{8-10}$ and avoiding postoperative continuation of antibiotic prophylaxis has been associated with a reduced risk of $C$ difficile infection. ${ }^{11}$ On the basis of a systematic review 


\section{Research in context}

\section{Evidence before this study}

Approximately one in six in-hospital prescriptions for antibiotics is for surgical antibiotic prophylaxis, which is frequently continued for several days after surgery. Although the effectiveness of appropriate surgical antibiotic prophylaxis to prevent surgical site infections in indicated procedures is well established, an increasing body of evidence suggests that a single preoperative dose of antibiotic, with repeat administration intraoperatively when indicated, might be as effective as a prolonged postoperative regimen. Prolonged exposure to antibiotics has been associated with antimicrobial resistance, Clostridioides difficile infection, and acute kidney injury. Across surgical subspecialties, many randomised controlled trials (RCTs) and some systematic reviews have investigated the benefit of prolonging antibiotic prophylaxis after surgery. In 2015, WHO did a systematic review and meta-analysis of the effect of antibiotic prophylaxis continuation on surgical site infection risk, and on the basis of the results, recommended against postoperative continuation of antibiotic prophylaxis; however, only a summary of the review was published. In 2018, a multicountry study in Europe and a global internet-based point prevalence study found that antibiotic prophylaxis is still routinely continued for several days after surgery. Additional trials became available after publication of the WHO guidelines, and some of the data included in the original WHO review might no longer be representative for current best practice standards of surgical antibiotic prophylaxis.

\section{Added value of this study}

This systematic review of 83 RCTs, of which 52 RCTs were included in the primary meta-analysis of studies that compared postoperative continuation of antibiotic prophylaxis with its immediate discontinuation, provides a comprehensive overview of all the available evidence on the practice of postoperative continuation of antibiotic prophylaxis across surgical subspecialties. Based on moderatecertainty evidence, we found no conclusive evidence that patients benefit from continued antibiotic prophylaxis after surgery in terms of the risk of surgical site infection. A prespecified subgroup analysis indicated that postoperative continuation of antibiotic prophylaxis is only effective in preventing surgical site infection when preoperative antibiotic prophylaxis is not timed adequately and not repeated intraoperatively according to the duration of the procedure. By contrast, when best practice standards of surgical antibiotic prophylaxis regarding timing and intraoperative repetition are applied, postoperative continuation of antibiotic prophylaxis has no effect on the risk of surgical site infection.

Implications of all the available evidence

Considering the possible adverse effects of surgical antibiotic prophylaxis, there is no basis for its postoperative continuation. Increased awareness and education about best practices are needed among patients and practitioners, which would encourage stewardship efforts among surgeons. Future research to further clarify the benefit of antibiotic continuation after surgery, if any, should monitor prespecified adverse events and standardise preoperative timing and intraoperative dose repetition according to evidence-based standardised criteria. and meta-analysis that included studies in a wide range of surgical subspecialties, WHO strongly recommended against postoperative continuation of antibiotic prophylaxis in their 2016 global guidelines for prevention of surgical site infections..$^{12}$ The US Centers for Disease Control and Prevention (CDC), the National Institute for Health and Care excellence (NICE), and other organisations made similar recommendations. ${ }^{5,13,14}$ Despite this advice, continuation of antibiotic prophylaxis after surgery is still a common practice worldwide. ${ }^{4} \mathrm{~A}$ global point prevalence study of 2015 data revealed that the percentage of patients who receive surgical antibiotic prophylaxis for more than 1 day after surgery ranged from $29.5 \%$ in developed countries in Europe to $92.5 \%$ in Africa. ${ }^{4}$ A scarcity of use monitoring and poor implementation of antimicrobial stewardship programmes might facilitate the continuation of this practice. ${ }^{15,16}$ However, low awareness of the existing evidence, and new, potentially contradicting evidence, might also contribute.

Only a summary of the systematic review done for the WHO recommendation was published. ${ }^{17}$ New evidence has continued to emerge with the publication of new randomised controlled trials (RCTs), ${ }^{18,19}$ and some of the data used in the initial review might no longer be representative of best practice standards for surgical antibiotic prophylaxis. ${ }^{5}$ Therefore, we did a systematic literature review and meta-analysis to update the evidence on which the 2016 WHO recommendation was based. We sought to reassess the effect of postoperative continuation of antibiotic prophylaxis on the incidence of surgical site infection compared with its postoperative discontinuation in patients undergoing surgical procedures.

\section{Methods}

\section{Search strategy and selection criteria}

The protocol for this systematic review and meta-analysis is registered with PROSPERO (CRD42017060829). This study is reported according to the PRISMA (Preferred Reporting Items for Systematic Reviews and Metaanalyses) statement. ${ }^{20}$

We searched MEDLINE (PubMed), Embase, CINAHL, CENTRAL, and WHO regional medical databases for studies published from Jan 1, 1990 to July 24, 2018. We searched combinations of the terms "surgical wound 
For the DrugBank database see https://www.drugbank.ca

See Online for appendix

For Google Translate see https://translate.google.com infection", "surgical site infection”, "SSI", "SSIs", "surgical infection", "post-operative wound infection", "postoperative wound infection", "antibiotic prophylaxis", "antimicrobial", "antibiotic", "prolong”, "duration”, "short”, "long”, "single dose", and "multi-dose". These terms were used according to the highly sensitive search strategy of Cochrane for identifying RCTs. ${ }^{21}$ The full search strategy is available in the appendix ( $p$ 1). We also searched reference lists of articles identified by our search for any relevant studies.

We included RCTs investigating the effect of postoperative continuation of antibiotic prophylaxis compared with its discontinuation on the incidence of surgical site infection in patients undergoing any surgical procedure with an indication for antibiotic prophylaxis. ${ }^{4}$ We excluded studies that compared regimens that also differed with regard to dose and drug used, studies that did not administer the first dose preoperatively by intravenous infusion, studies on dirty procedures according to CDC wound classification, ${ }^{22}$ or established infections for which antibiotic use was classified as treatment, observational and preclinical studies, and duplicate studies. We limited eligibility to studies published from 1990 onwards, as in the WHO analysis, because infection prevention practices before 1990 differed substantially from current practices. We applied no restrictions regarding the definition of outcomes, length of follow up, or language of publication.

Two authors (QJJB and SWdJ) independently screened the titles and abstracts for eligibility. If a title and abstract indicated potential eligibility, the full-text article was obtained. To avoid language bias, articles published in languages other than English were translated by authors proficient in the language or, when unavailable, by the online multilingual translation service Google Translate. ${ }^{23}$ Any disagreements were resolved by discussion between the two authors or, when necessary, via consultation with the senior author (MAB).

\section{Data extraction}

Two authors (QJJB and SWdJ) independently reviewed each eligible article and extracted relevant data using a prespecified data extraction form. Data collection covered design; publication date; number of participants (and proportions of male and female participants); mean participant age; type of surgery; contamination according to $\mathrm{CDC}$ wound classification;22 outcome definition and follow-up; dosage and regimen of antibiotics in the intervention and control groups, including timing of the preoperative dose and intraoperative repeat administration when indicated; aggregate data on study outcome; resource use expressed in costs; and adverse events. If data were not given in a paper, we contacted authors by email, or post if email was not available, for detailed information on timing of the first dose of antibiotics, surgery duration, intraoperative repeat administration, adverse events, and antibiotics used. To uniformly assess if repeat administration was indicated, we obtained information on drug half-life from the clinical practice guidelines of the American Society of Health-System Pharmacists on antimicrobial prophylaxis in surgery, and from the comprehensive DrugBank database for bioinformatics and cheminformatics (version 5.0). ${ }^{24}$ When insufficient information could be retrieved on timing of the preoperative dose or intraoperative repeat administration, we assumed these variables were not standardised during the study, rather than excluding the study.

\section{Risk of bias assessment}

Two authors (QB and SWdJ) independently assessed the risk of bias of included studies using the Cochrane Collaboration's tool for assessing risk of bias in RCTs. ${ }^{2}$ The exact criteria used to determine risk of bias are listed in the appendix (p 2). Conflicts were resolved by discussion between the two authors or after consultation with the senior author. Results were displayed in summary figures generated by Review Manager (version 5.3). The possibility of publication bias was visually assessed with a contourenhanced funnel plot. ${ }^{25}$

\section{Data analysis}

Trials of any surgical procedure that compared postoperative continuation of antibiotic prophylaxis of any duration with its immediate discontinuation (ie, no doses after the preoperative dose and any intraoperative administration) were included in the primary analysis. The primary outcome was the effect of postoperative continuation versus immediate discontinuation of surgical antibiotic prophylaxis on the occurrence of surgical site infection. Secondary analyses were done comparing postoperative regimens of different durations. Additional outcomes of interest were frequency and type of adverse events, as defined in each paper according to the authors' discretion, and resource use. We calculated summary relative risks (RRs) with corresponding 95\% CIs using a random effects model (DerSimonian and Laird), thus taking into account statistical heterogeneity. ${ }^{26}$ We assessed heterogeneity with the $\chi^{2}$ test, and expressed the ratio of true heterogeneity to total variation in observed effects using the $I^{2}$ statistic. The extent of heterogeneity was evaluated with the $\tau^{2}$ statistic. We used the empirical distribution for pharmacological versus pharmacological comparisons on subjective outcomes to characterise the amount of heterogeneity as low, moderate, or high using the first and third quantiles of their empirical dis tributions. $^{27}$

Current best practice standards for surgical antibiotic prophylaxis are described in the American Society of Health-System Pharmacists clinical practice guidelines on antimicrobial prophylaxis in surgery. ${ }^{5}$ We accounted for these standards in prespecified subgroup analyses of the main outcome for studies that standardised: 1) timing of the first preoperative dose within 60 min before incision; 2) repeat administration when the procedure duration exceeded two times the half-life of the antibiotic used; and 3) adherence to current best practice standards 
meeting both of these conditions. To investigate potential procedure-specific effects, we also did post-hoc exploratory subgroup analyses by procedure type. For each subgroup analysis, we used random-effects meta-regression to investigate the association of subgroup characteristics with the intervention effect. ${ }^{28}$ The proportion of variance explained by each subgroup was calculated by examining the change from the main analysis in $\tau^{2},{ }^{29}$ and expressed as percentage of heterogeneity variance explained:

$\left(\frac{\tau^{2} \text { meta-analysis (overall) }-\tau^{2} \text { meta-regression }}{\tau^{2} \text { meta-analysis (overall) }}\right)$

Statistical analyses were done in Stata (version 15.0).

We used the Grading of Recommendations Assessment, Development and Evaluation (GRADE) methodology with GRADE Pro software to judge the certainty of retrieved evidence. ${ }^{30}$ Predefined subgroups with a strong association with the intervention effect ( $\mathrm{p}$ value for metaregression, <0.05) were graded individually. Optimal information size, defined as the number of participants needed for a single adequately powered trial, was calculated assuming a type I error $(\alpha)$ of $0 \cdot 05$, a type II error $(\beta)$ of $0 \cdot 2$, and an RR reduction of $25 \% .^{30}$ If a confidence interval did not exclude appreciable benefit of surgical antibiotic prophylaxis continuation, defined as an RR reduction of $25 \%$, the certainty of evidence was downgraded regardless of the optimal information size. ${ }^{30}$

\section{Role of the funding source}

There was no funding for this study. The corresponding author had full access to all the data in the study and had final responsibility for the decision to submit for publication.

\section{Results}

Our database search retrieved 3238 potentially relevant records, and 24 additional records were identified from the reference lists of retrieved records. We assessed 147 full-text publications for eligibility; 83 RCTs were critically appraised and included in the meta-analysis. The selection procedure is summarised in figure 1 . Reasons for exclusion after full-text assessment are described in the appendix (pp 3,4).

Study characteristics of the included RCTs are listed in the appendix (pp 5-11). In total, 24318 participants were included in the 83 RCTs comparing different postoperative antibiotic prophylaxis regimens, with surgical site infection as an outcome. Source countries were Argentina, Australia, Canada, China, Brazil, France, Germany, Hong Kong, India, Iran, Israel, Italy, Japan, the Netherlands, Pakistan, Romania, Saudi Arabia, South Korea, Spain, Sweden, Switzerland, Taiwan, Tanzania, Thailand, the UK, and the USA.

The mean age of included patients was 47.8 years (SD 16.2), and ages ranged from 8 years to 77 years. All but one study focused primarily on adult participants (aged $\geq 18$ years). 19 studies included children. The mean percentage of female patients included was $47.6 \%$ (SD 0.2), and the proportion ranged from $10 \%$ (one RCT with three female participants of 30 total participants) to $100 \%$ (one RCT with 500 female participants). Procedures were diverse and represented gastrointestinal, cardiac, thoracic, head and neck, gynaecological, obstetrics, trauma, orthopaedics, and maxillofacial surgery. 66 trials were single-centre studies.

52 RCTs involving 19273 participants compared postoperative continuation of antibiotic prophylaxis, varying from one postoperative administration to 5 days of postoperative continuation, with its immediate postoperative discontinuation. 33 RCTs involving 5416 participants compared postoperative antibiotic prophylaxis regimens of different durations. Two RCTs had several study groups and provided data on multiple comparisons (Rajabi-Mashhadi et al, 2012, and Mui et al, 2005), which were included in the primary analysis and comparison of prophylaxis for greater than $24 \mathrm{~h}$ versus $24 \mathrm{~h}$ or less after surgery (figure 1).

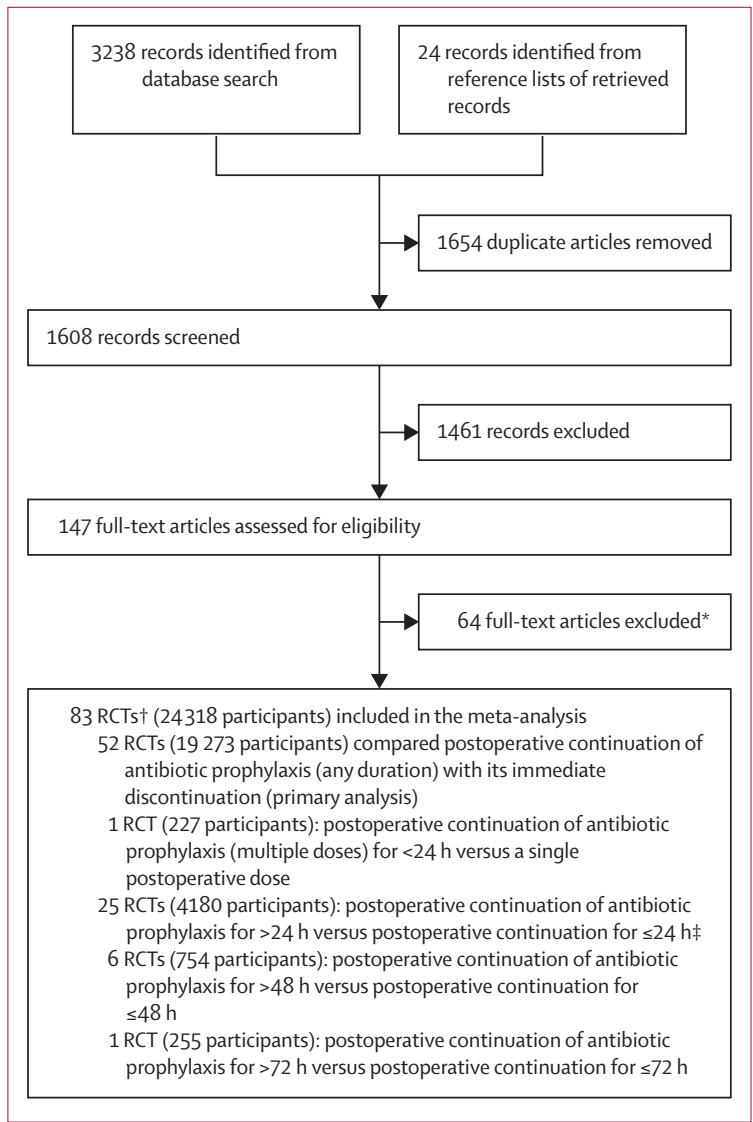

Figure 1: Study selection process

RCT=randomised controlled trial. *Reasons for exclusion after full-text assessment are shown in the appendix (pp 3, 4). †Two RCTs had several study groups and provided data on multiple comparisons (included in the primary analysis and $>24 \mathrm{~h} v \mathrm{~s} \leq 24 \mathrm{~h}$ comparison). $\neq$ Five trials assessed a single postoperative dose in their $\leq 24 \mathrm{~h}$ group.
For the GRADE Pro software see http://gradepro.org/ 
Timing of the first preoperative dose of antibiotics was standardised as within $60 \mathrm{~min}$ before the first incision in 57 RCTs. 46 RCTs had standardised intraoperative repeat administration or had procedure durations that did not exceed two half-lives of the antibiotic used. Best practice standards (ie, correct timing before incision and repeat administration during surgery when indicated) were adhered to in 24 RCTs. In 26 RCTs, surgical site infection was described as the current CDC definition. ${ }^{22}$ The other 57 studies used descriptions ranging from purulent discharge to extensive, field-specific descriptions of the clinical manifestation of surgical site infection. 24 studies reported adverse events and six studies described resource use. All outcome definitions are listed in the appendix (p 12).

Our primary meta-analysis of 52 RCTs showed an indication, but not conclusive evidence, of a benefit of postoperative continuation of antibiotic prophylaxis for the prevention of surgical site infection compared with its immediate postoperative discontinuation (RR 0.89 [95\% CI 0.79-1.00]; table 1, figure 2). Heterogeneity in effect size between studies was low $\left(\tau^{2}=0 \cdot 001, \chi^{2} p=0 \cdot 46\right.$, $I^{2}=0 \cdot 7 \%$ ). Our subgroup analyses and meta-regressions indicated that compliance with best practice standards for surgical antibiotic prophylaxis significantly modified the association between postoperative continuation of antibiotic prophylaxis and the incidence of surgical site infection (table 1, figure 2). Only in 27 trials that were not compliant with best practice standards (ie, first preoperative dose given >60 min before incision and repeat administration not standardised when procedure duration exceeded two times the half-life of the antibiotic) did continuation of antibiotic prophylaxis after surgery prevent surgical site infection compared with its immediate discontinuation (RR 0.79 [0.67-0.94]); corresponding heterogeneity was moderate $\left(\tau^{2}=0 \cdot 019\right.$, $\left.\chi^{2} \mathrm{p}=0 \cdot 31, I^{2}=10 \cdot 3 \%\right)$. When the analysis was restricted to 24 trials that met best practice standards of surgical antibiotic prophylaxis regarding timing and repeat administration, there was no benefit of postoperative continuation of antibiotic prophylaxis $(1 \cdot 04$ [0 . 85-1.27]; subgroup $\mathrm{p}=0 \cdot 048,100 \%$ variance explained). In trials that met best practice standards, corresponding heterogeneity in effect size was low $\left(\tau^{2}<0 \cdot 001, \chi^{2} p=0 \cdot 78\right.$, $\left.I^{2}<0 \cdot 1 \%\right)$.

Adequate timing or repeat administration alone did not affect the effect estimate (table 1). In an exploratory subgroup analysis and meta-regression, we found some evidence that postoperative continuation of antibiotic prophylaxis might reduce the risk of surgical site infection compared with its immediate discontinuation in some surgical subspecialties_-specifically, maxillofacial surgery and cardiac surgery (appendix pp 13, 14). However, no studies standardised adherence to best practice standards in the cardiac surgery subgroup, and only three studies standardised adherence for maxillofacial surgery. The results of all exploratory subgroup analyses are presented in the appendix (pp 13, 14). The remaining meta-analyses

\begin{tabular}{|c|c|c|c|c|c|c|c|c|}
\hline & $\begin{array}{l}\text { Number of } \\
\text { studies }\end{array}$ & $\begin{array}{l}\text { SSIs/total } \\
\text { participants in } \\
\text { longer regimen }\end{array}$ & $\begin{array}{l}\text { SSIs/total } \\
\text { participants in } \\
\text { shorter regimen }\end{array}$ & $\begin{array}{l}\text { Relative risk } \\
(95 \% \mathrm{Cl})\end{array}$ & $\begin{array}{l}\tau^{2} \text { (meta- } \\
\text { analysis) }\end{array}$ & $\begin{array}{l}\tau^{2}(\text { meta- } \\
\text { regression) }\end{array}$ & $\begin{array}{l}p \text { value for } \\
\text { subgroup } \\
\text { differences }\end{array}$ & $\begin{array}{l}\% \text { of } \\
\text { heterogeneity } \\
\text { variance } \\
\text { explained }\end{array}$ \\
\hline \multicolumn{9}{|c|}{ Postoperative continuation vs immediate discontinuation of SAP (primary analysis) } \\
\hline Overall & 52 & $492 / 9726(5 \cdot 1 \%)$ & $550 / 9547(5 \cdot 8 \%)$ & $0.89(0.79-1.00)$ & 0.001 & NA & NA & NA \\
\hline $\begin{array}{l}\text { Timing of first dose specified and within } 60 \text { min before } \\
\text { surgery }\end{array}$ & .. &.. & .. & .. &.$\cdot$ & 0 & 0.13 & $100 \%$ \\
\hline Yes & 33 & $303 / 6249(4 \cdot 8 \%)$ & $314 / 6151(5 \cdot 1 \%)$ & $0.96(0.82-1.12)$ & $<0.001$ & .. & .. & .. \\
\hline No & 19 & $189 / 3477(5 \cdot 4 \%)$ & $236 / 3396(6 \cdot 9 \%)$ & $0.77(0.61-0.96)$ & 0.033 & .. & .. & .. \\
\hline $\begin{array}{l}\text { Intraoperative repeat administration specified when } \\
\text { indicated }\end{array}$ & .. & .. & .. & .. & .. & 0.005 & 0.88 & $0 \%$ \\
\hline Yes & 34 & $265 / 6126(4 \cdot 3 \%)$ & $288 / 5944(4 \cdot 8 \%)$ & $0.89(0.76-1.05)$ & $<0.001$ & .. & .. & .. \\
\hline No & 18 & $227 / 3600(6 \cdot 3 \%)$ & $262 / 3603(7 \cdot 3 \%)$ & $0.86(0.70-1.05)$ & 0.021 & .. & .. & .. \\
\hline Adherence to current best practice standards of SAP* & .. & .. & .. & .. & .. & 0 & 0.048 & $100 \%$ \\
\hline Yes & 24 & $196 / 4648(4 \cdot 2 \%)$ & $186 / 4552(4 \cdot 1 \%)$ & $1.04(0.85-1.27)$ & $<0.001$ & .. & .. & .. \\
\hline No & 28 & $296 / 5078(5 \cdot 8 \%)$ & $364 / 4995(7 \cdot 3 \%)$ & $0.79(0.67-0.94)$ & 0.019 & .. & .. & .. \\
\hline \multicolumn{9}{|l|}{ Secondary analyses } \\
\hline $\begin{array}{l}\text { Postoperative continuation (multiple doses) for } \\
<24 \mathrm{~h} \text { vs a single dose after surgery }\end{array}$ & 1 & $44 / 113(38.9 \%)$ & $39 / 114(34 \cdot 2 \%)$ & $0.82(0.57-1.40)$ & NA & NA & NA & NA \\
\hline Postoperative continuation for $>24 \mathrm{~h}$ vs for $\leq 24 \mathrm{~h} \dagger$ & 25 & $170 / 2081(8 \cdot 2 \%)$ & $191 / 2099(9 \cdot 1 \%)$ & $0.93(0.76-1.13)$ & $<0.001$ & NA & NA & NA \\
\hline Postoperative continuation for $>48 \mathrm{~h}$ vs for $\leq 48 \mathrm{~h}$ & 6 & $48 / 372(12 \cdot 9 \%)$ & $35 / 382(9 \cdot 2 \%)$ & $1.35(0.89-2.03)$ & $<0.001$ & NA & NA & NA \\
\hline Postoperative continuation for $>72 \mathrm{~h}$ vs for $\leq 72 \mathrm{~h}$ & 1 & $3 / 125(2 \cdot 4 \%)$ & $4 / 130(3 \cdot 1 \%)$ & $0.61(0.14-2 \cdot 63)$ & NA & NA & NA & NA \\
\hline
\end{tabular}




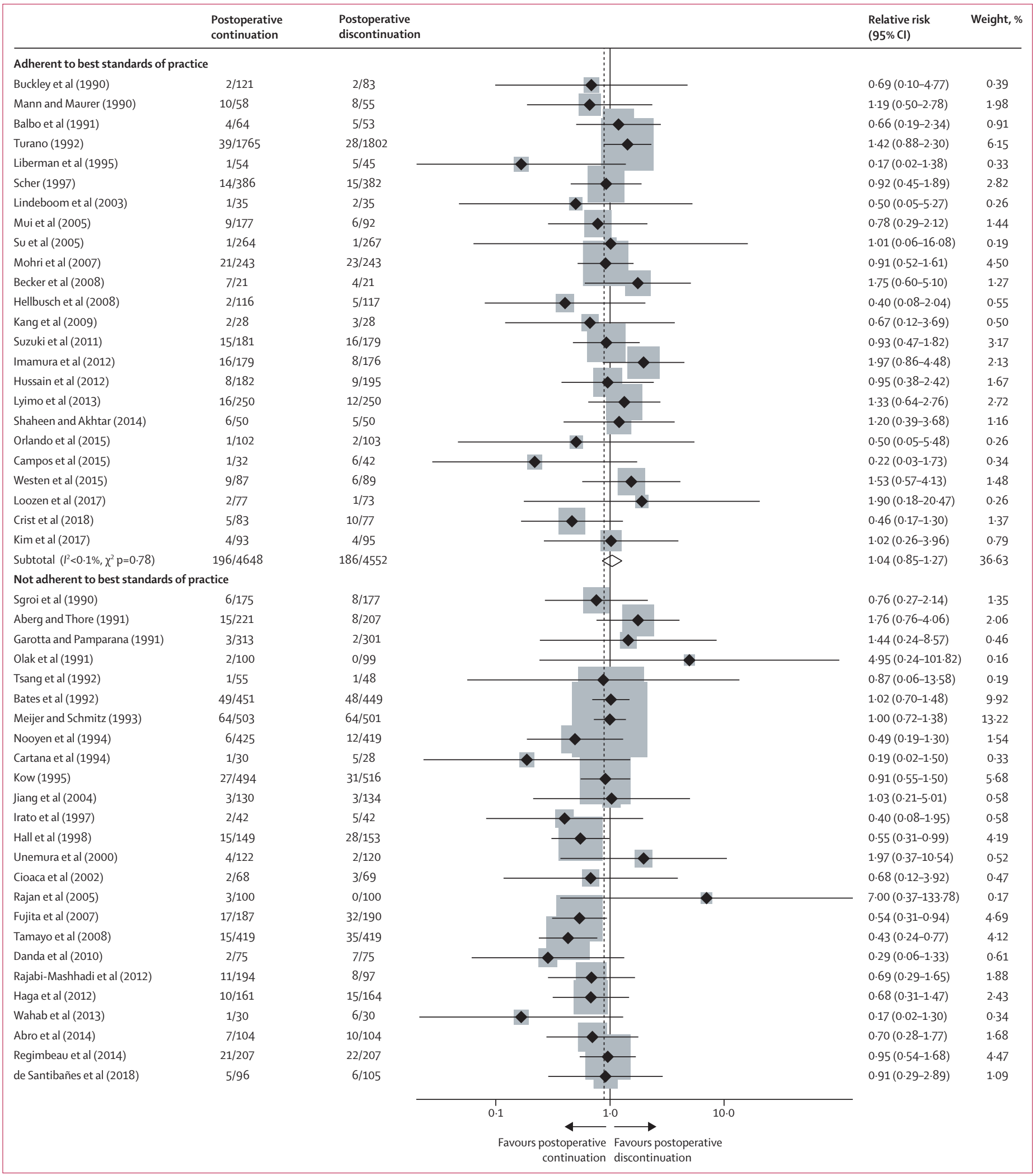




\begin{tabular}{|c|c|c|c|c|c|}
\hline & $\begin{array}{l}\text { Postoperative } \\
\text { continuation }\end{array}$ & $\begin{array}{l}\text { Postoperative } \\
\text { discontinuation }\end{array}$ & & $\begin{array}{l}\text { Relative risk } \\
(95 \% \mathrm{Cl})\end{array}$ & Weight, $\%$ \\
\hline Sadraei-Moosavi et al (2017) & $1 / 76$ & $1 / 76$ & 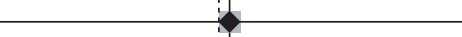 & $1.00(0.06-15 \cdot 70)$ & 0.19 \\
\hline Chauhan et al (2018) & $3 / 98$ & $2 / 112$ & $\begin{array}{l:c} & \end{array}$ & $1.71(0.29-10.05)$ & 0.46 \\
\hline Maier and Strutz (1992) & $0 / 53$ & $0 / 53$ & 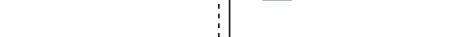 & NA & 0.00 \\
\hline \multirow[t]{2}{*}{ Subtotal $\left(I^{2}=10 \cdot 3 \%, \chi^{2} p=0.31\right)$} & $296 / 5078$ & $364 / 4995$ & $\Delta$ & $0.79(0.67-0.94)$ & 63.37 \\
\hline & & & & & $100 \cdot 00$ \\
\hline \multirow[t]{2}{*}{ Overall $\left(I^{2}=0.7 \%, \chi^{2} \mathrm{p}=0.46\right)$} & $492 / 9726$ & $550 / 9547$ & i) & $0.89(0.79-1.00)$ & \\
\hline & & & Favours postoperative $\underset{\begin{array}{c}\text { Favours postoperative } \\
\text { continuation }\end{array}}{\longrightarrow}$ & & \\
\hline
\end{tabular}

Figure 2: Relative risk of surgical site infection with postoperative continuation versus immediate discontinuation of antibiotic prophylaxis

The figure shows the pooled estimate from 52 RCTs that compared the effect of postoperative continuation of antibiotic prophylaxis with its immediate discontinuation on the risk of surgical site

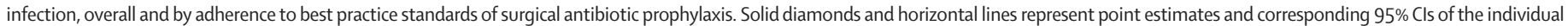
studies. Shading around each point represents study weight. Transparent diamonds represent the overall estimate and $95 \% \mathrm{Cl}$. Full citations are in the appendix (pp 30-36). RR=relative risk. $\mathrm{NA}=$ not available.

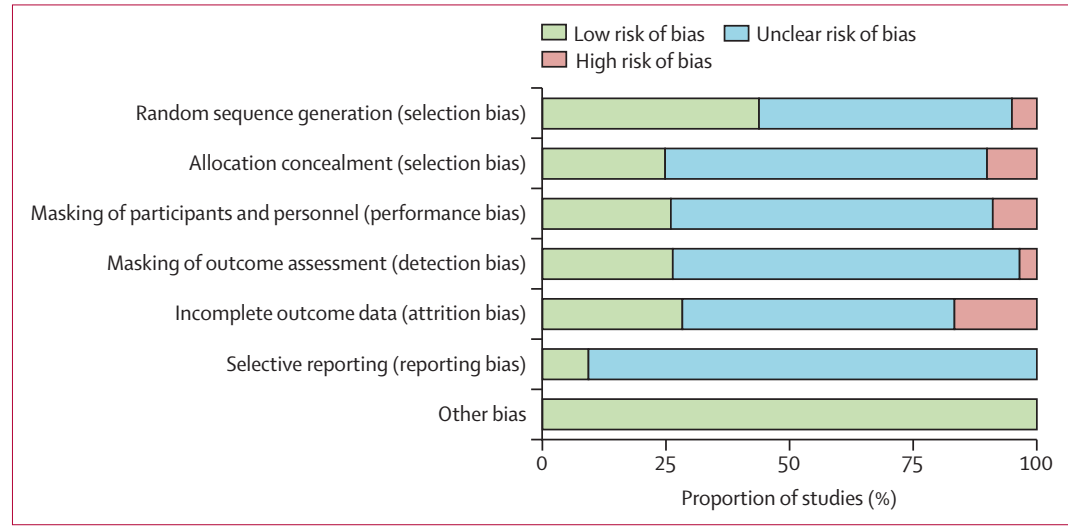

Figure 3: Risk of bias in the included studies

Risk was defined according to the Cochrane Collaboration's tool for assessing risk of bias in RCTs (full criteria in appendix $\mathrm{p} 2)^{21}$

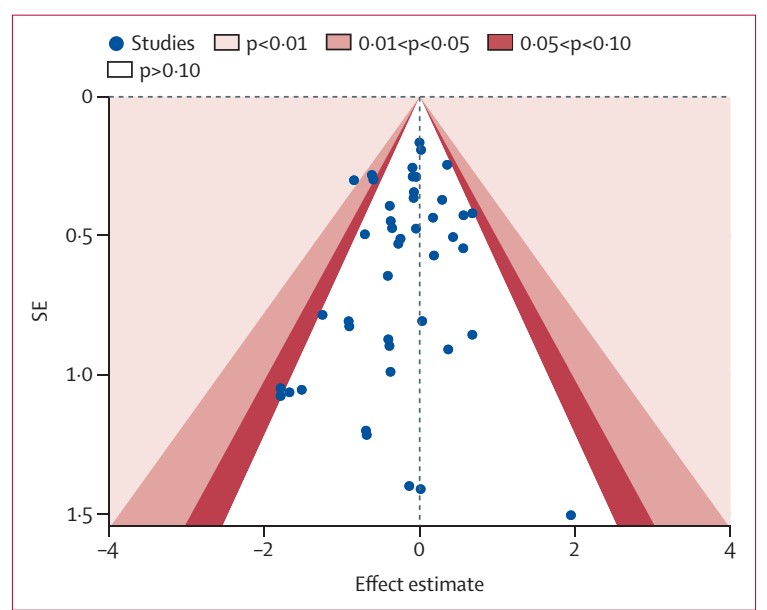

Figure 4: Funnel plot for the primary analysis of postoperative continuation versus immediate discontinuation of antibiotic prophylaxis Effect estimates of the different studies (blue dots) are shown against their precision (standard error). Contours represent levels of statistical significance, derived from the two axes of the plot. ${ }^{25}$ Asymmetry across the vertical midline, representing the overall effect estimate of the meta-analysis, indicates publication bias. The plot shows a symmetrical distribution and no indication of publication bias. comparing continuation of antibiotic prophylaxis after surgery for different durations did not provide conclusive evidence that surgical site infections were prevented by longer continuation of postoperative antibiotic prophylaxis (table 1). Forest plots of the individual meta-analyses are presented in the appendix (pp 15-20).

24 studies (17 included in the primary analysis) described possible harmful effects or adverse events related to surgical antibiotic prophylaxis (appendix p 21). Of these, 18 studies could not attribute adverse events to antibiotic use in both the intervention and control groups. The remaining six studies reported increased adverse events in the groups with prolonged regimens. Of these, one study reported increased cases of $C$ difficile infection in the prolonged postoperative continuation group. The other studies reported an increased frequency of rash and pruritus, erythema, phlebitis, hypotension, gastrointestinal disturbance (including nausea and diarrhoea), and unspecified local and systemic side-effects with postoperative continuation of antibiotic prophylaxis. No study reported on antimicrobial resistance. Owing to heterogeneity between studies in the comparisons made and the outcomes measured, no meta-analysis could be done of adverse effects.

Five studies (four included in our primary analysis) addressed cost-effectiveness and reported a cost increase associated with longer antibiotic prophylaxis regimens, in some cases as a result of treatment for side-effects and hospitalisation time in addition to prophylaxis treatment, which varied from US\$36.90 to $\$ 78.95$ (appendix p 22). None of these studies calculated costs associated with the emergence of antimicrobial resistance. All five studies were done in high-income countries (Australia, Italy, Taiwan, and the USA).

A summary of our risk of bias evaluations with the Cochrane tool is presented in figure 3 , and the full evaluations are provided in the appendix (pp 23-28). Many assessments had unclear risk of bias, and some assessments were of high risk of bias. No funnel plot asymmetry was detected for the comparison of continued postoperative antibiotic prophylaxis (any duration) with 


\begin{tabular}{|c|c|c|c|c|c|c|c|c|}
\hline & \multirow{2}{*}{$\begin{array}{l}\text { Number } \\
\text { of } \\
\text { studies }\end{array}$} & \multicolumn{6}{|c|}{ Certainty assessment } & \multirow{2}{*}{$\begin{array}{l}\text { Certainty } \\
\text { of } \\
\text { evidence }\end{array}$} \\
\hline & & Study design & Risk of bias & Inconsistency & Indirectness & Imprecision & $\begin{array}{l}\text { Other } \\
\text { considerations }\end{array}$ & \\
\hline $\begin{array}{l}\text { Postoperative continuation vs immediate } \\
\text { discontinuation of SAP (primary analysis) }\end{array}$ & 52 & RCTs & Serious* ${ }^{*}$ & Not serious & Not serious & Not serious (OIS: 3629 per group) & None & Moderate \\
\hline Adhered to best practice standards of SAP & 24 & RCTs & Serious* & Not serious & Not serious & Not serious (OIS: 5185 per group) & None & Moderate \\
\hline $\begin{array}{l}\text { Did not adhere to best practice standards of } \\
\text { SAP }\end{array}$ & 28 & RCTs & Serious* & Not serious & Not serious & Not serious (OIS: 2823 per group) & None & Moderate \\
\hline $\begin{array}{l}\text { Postoperative continuation (multiple doses) } \\
\text { for }<24 \mathrm{~h} \text { vs a single dose after surgery } \\
\text { (secondary analysis) }\end{array}$ & 1 & $\mathrm{RCT}$ & Serious* & Not serious & Not serious & Serious $\ddagger$ (OIS: 444 per group) & None & Low \\
\hline $\begin{array}{l}\text { Postoperative continuation for }>24 \mathrm{~h} v \mathrm{vs} \leq 24 \mathrm{~h} \\
\text { (secondary analysis) }\end{array}$ & 25 & RCTs & Serious* & Not serious & Not serious & Serioust (OIS: 2168 per group) & None & Low \\
\hline $\begin{array}{l}\text { Postoperative continuation for }>48 \mathrm{~h} v \mathrm{vs} \leq 48 \mathrm{~h} \\
\text { (secondary analysis) }\end{array}$ & 6 & RCTs & Serious* & Not serious & Not serious & Serious† (OIS: 2515 per group) & None & Low \\
\hline $\begin{array}{l}\text { Postoperative continuation for }>72 \mathrm{~h} v \mathrm{~s} \leq 72 \mathrm{~h} \\
\text { (secondary analysis) }\end{array}$ & 1 & $\mathrm{RCT}$ & Serious* & Not serious & Not serious & Serioust‡ (OIS: 6950 per group) & None & Low \\
\hline
\end{tabular}

its immediate discontinuation (figure 4), or for the comparison of postoperative continuation for more than $24 \mathrm{~h}$ with postoperative continuation for $24 \mathrm{~h}$ or less (appendix p 29). There were too few data for the other three comparisons to allow adequate evaluation of the funnel plots.

An evidence table of our GRADE assessments is presented in table 2. All included studies were RCTs, and thus the starting certainty of the evidence for each comparison was high. For the primary analysis, the certainty of evidence was downgraded to moderate because of serious risk of bias. One subgroup analysis, adherence to current best practice standards, was graded individually because of its strong association with the intervention effect. For both subgroups, the certainty of evidence was downgraded to moderate because of serious risk of bias. The certainty of evidence for the remaining analyses, comparing postoperative regimens of different durations, was downgraded to low in each comparison because of serious risk of bias and imprecision.

\section{Discussion}

Moderate-certainty evidence from a meta-analysis of 52 RCTs involving 19273 participants showed no conclusive evidence for a benefit of postoperative continuation of antibiotic prophylaxis in reducing the incidence of surgical site infection compared with its immediate discontinuation. Similarly, low-certainty evidence from comparisons of postoperative regimens of different durations showed no conclusive evidence of a benefit of prolonged regimens. Our subgroup analysis showed that the effectiveness of postoperative discontinuation of antibiotic prophylaxis depend on the appropriateness of the surgical antibiotic prophylaxis practices. When best practices (ie, timely administration of the first dose and repeat administration when indicated according to the procedure duration) were applied, moderate-certainty evidence showed no benefit of postoperative continuation of antibiotic prophylaxis in reducing surgical site infections compared with its discontinuation. Moderate-certainty evidence showed that postoperative continuation of antibiotic prophylaxis was effective only when these standards were not met. Some evidence from our exploratory analysis indicated that postoperative continuation of antibiotic prophylaxis might reduce the risk of surgical site infection associated with maxillofacial and cardiac surgery; however, no studies that adhered to best practice standards were available for the cardiac surgery subgroup, and very few were available for the maxillofacial surgery subgroup. When costs and adverse events were reported, postoperative continuation appeared to increase costs and lead to more adverse events.

Our findings are in line with the initial review that supported the WHO recommendation against postoperative continuation of antibiotic prophylaxis, which found an odds ratio of 0.89 (95\% CI $0 \cdot 77-1.03)$ for the likelihood of SSI with postoperative continuation. ${ }^{12}$ In comparison with this initial WHO meta-analysis, the confidence interval resulting from the present metaanalysis has narrowed slightly as further data has accrued, but the point estimate remains unchanged. Although a small benefit is indicated by both estimates, the confidence intervals include unity, and do not indicate appreciable benefit with regard to prevention of surgical site infection.

Antibiotic use is associated with important adverse effects in a duration-dependent manner. ${ }^{8-10}$ In turn, these 
adverse effects are associated with a substantial economic burden that adds to additional acquisition and administration costs related to postoperative continuation of antibiotic prophylaxis. ${ }^{31-33}$ Such costs are particularly consequential in developing countries with limited resources, where this practice is most prevalent. ${ }^{4}$ In many settings worldwide, postoperative continuation of antibiotic prophylaxis is often used to compensate for an absence of routine best practices and gaps in other infection prevention measures; this conflicts with the basic principles of antibiotic stewardship. ${ }^{34}$

Most guidelines issued before the WHO guidelines on surgical site infection prevention recommend prolongation of surgical antibiotic prophylaxis to a maximum of $24-48 \mathrm{~h}$, but they were not based on rigorous evaluation of the existing evidence by systematic review., ${ }^{5,13}$ Other systematic reviews that have addressed this issue have focused on one specific procedure, limiting power and generalisability, and included studies that compared regimens that differed in terms of dosage or drug, in addition to the duration of administration. ${ }^{35,36}$ Indications that postoperative antibiotic prophylaxis might have no added value arose as early as the $1960 \mathrm{~s} .{ }^{37}$ Since then, routine postoperative continuation of antibiotic prophylaxis has persisted, ${ }^{38}$ even with increasing concerns about antimicrobial resistance and other adverse effects. ${ }^{1,2}$ Despite the recent WHO guidelines, antibiotic prophylaxis is still routinely continued for several days after surgery. ${ }^{4}$

An important limitation of our analysis is that only $24(46 \%)$ of 52 included studies standardised current best practices of surgical antibiotic prophylaxis. Any beneficial effect identified in the overall estimate, therefore, could reflect compensation of poor preoperative timing or lack of repeat administration when indicated, and thus overestimate the true effect of surgical antibiotic prophylaxis continuation. To account for this bias, we did a prespecified subgroup analysis that accounted for standardisation of best practices of surgical antibiotic prophylaxis. However, we could only extract aggregate data from all publications and did not have individual patient data, thus limiting the granularity of the data and the possibilities for detailed subgroup analysis..$^{39}$ An exploratory subgroup analysis into the effect of postoperative continuation of antibiotic prophylaxis in certain surgical subspecialties was limited by a large number of subgroups, and consequently small numbers per subgroup. These characteristics lead to a high risk of false-positive results, and the analysis should be interpreted with caution.

Strengths of this study include the broad inclusion criteria and relevant exclusion criteria. Data were from 28 different countries and represented adult and paediatric populations and a variety of surgical procedures, suggesting broad generalisability. Additionally, the exclusion of studies of regimens that also differed with regard to dose and drug used, or studies that concerned antibiotic treatment rather than prophylaxis, ensured the elimination of important sources of bias. However, poor reporting of surgical trials, as previously noted, ${ }^{40}$ was also an issue in this analysis. This limitation is in part due to an absence of reporting standards before $2010 .{ }^{41}$ As a result, a considerable proportion of the risk of bias was unclear, and important information on timing of the first dose of antibiotics, procedure duration, intraoperative repeat administration, adverse events, or antibiotic used was frequently missing. We contacted the authors of such studies to request further information, but not all replied to our request. Consequently, we had to assume best practices were not in place in some studies because the required information could not be attained. The subgroup analysis might therefore be contaminated by reporting standards and responsiveness of the corresponding author and should be interpreted with caution. More than half (57 [69\%]) of the 83 included RCTs used definitions of surgical site infection that differed from the widely accepted CDC criteria. ${ }^{22}$ In most cases, this divergence in definition is again attributable to the time of publication. The current CDC definition was published in 1992, and thus many alternatives, including preceding versions of the CDC definition, were in wider use throughout the 1990s. Such differences in outcome definition challenge interpretation of the clinical importance of our findings and their comparison with the results of future studies. However, an important aim of this study was to consider all available evidence, and previous evidence shows that alternative systems to define surgical site infections can provide information similar to that captured by the CDC criteria. ${ }^{42}$ Finally, costs and adverse events were poorly reported, if at all, and no meaningful meta-analyses could be done to assess these outcomes.

We found no conclusive evidence for a benefit of postoperative continuation of antibiotic prophylaxis compared with its discontinuation in reducing the incidence of surgical site infection. Notably, when best practices for surgical antibiotic prophylaxis were followed, postoperative continuation did not provide any additional benefit in preventing surgical site infection. Our findings support WHO recommendations against the practice of continuing surgical antibiotic prophylaxis postoperatively. Considering the associated adverse effects-in particular, antimicrobial resistance-this prevalent practice has no basis. Increased awareness and education are warranted among both health-care professionals and patients, especially by prioritising stewardship efforts among surgeons and anaesthetists and insisting on other infection prevention measures in addition to surgical antibiotic prophylaxis. Future research to clarify the benefit of continuation of antibiotic prophylaxis beyond surgery, if any, should prespecify monitoring of adverse events, provide detailed data on costs, and standardise preoperative timing and intraoperative repeat administration of antibiotics.

Contributors

SWdJ and MAB designed the study. SWdJ and Q JJB screened records, extracted data, and assessed risk of bias. SWdJ did the statistical analysis 
SWdJ, ME, GS, BA, PED, JSS, Q JJB, and MAB analysed and interpreted the data. SWdJ and Q JJB drafted the manuscript. All authors provided critical conceptual input, interpreted the data analysis, and critically revised the manuscript.

\section{Declaration of interests}

PED reports personal fees from Destiny Pharma, outside of the submitted work. MAB reports grants, invited speaker fees, and advisory board fees from KCI Medical and Ethicon, grants from New Compliance and Mylan, and speaker fees from Bard Medical, Gore, and Smith and Nephew, outside of the submitted work. GS reports attending meetings with Biogen and Merck, outside of the submitted work. All other authors declare no competing interests.

\section{Acknowledgments}

We thank F S van Etten-Jamaludin (University of Amsterdam, Amsterdam, Netherlands) for her advice on the search strategy, and all the investigators who responded to our requests for data and clarification. The authors alone are responsible for the views expressed in this Article, and they do not necessarily represent the views, decisions, or policies of the institutions with which they are affiliated. WHO takes no responsibility for the information provided or the views expressed in this paper.

\section{References}

1 Laxminarayan R, Matsoso P, Pant S, et al. Access to effective antimicrobials: a worldwide challenge. Lancet 2016 387: 168-75.

2 McDonald LC, Gerding DN, Johnson S, et al. Clinical practice guidelines for Clostridium difficile infection in adults and children: 2017 update by the Infectious Diseases Society of America (IDSA) and Society for Healthcare Epidemiology of America (SHEA). Clin Infect Dis 2018; 66: e1-48.

3 Plachouras D, Kärki T, Hansen S, et al. Antimicrobial use in European acute care hospitals: results from the second point prevalence survey (PPS) of healthcare-associated infections and antimicrobial use, 2016 to 2017. Euro Surveill 2018; 23: 1800393.

4 Versporten A, Zarb P, Caniaux I, et al. Antimicrobial consumption and resistance in adult hospital inpatients in 53 countries: results of an internet-based global point prevalence survey. Lancet Glob Health 2018; 6: e619-29.

5 Bratzler DW, Dellinger EP, Olsen KM, et al. Clinical practice guidelines for antimicrobial prophylaxis in surgery. Am J Health Syst Pharm 2013; 70: 195-283.

6 Regimbeau JM, Fuks D, Pautrat K, et al. Effect of postoperative antibiotic administration on postoperative infection following cholecystectomy for acute calculous cholecystitis: a randomized clinical trial. JAMA 2014; 312: 145-54.

7 Imamura H, Kurokawa Y, Tsujinaka T, et al. Intraoperative versus extended antimicrobial prophylaxis after gastric cancer surgery: a phase 3, open-label, randomised controlled, non-inferiority trial. Lancet Infect Dis 2012; 12: 381-87.

8 Stevens V, Dumyati G, Fine LS, Fisher SG, van Wijngaarden E. Cumulative antibiotic exposures over time and the risk of Clostridium difficile infection. Clin Infect Dis 2011; 53: 42-48.

9 Branch-Elliman W, O’Brien W, Strymish J, Itani K, Wyatt C Gupta K. Association of duration and type of surgical prophylaxis with antimicrobial-associated adverse events. JAMA Surg 2019; 154: 590-98.

10 Harbarth S, Samore MH, Lichtenberg D, Carmeli Y. Prolonged antibiotic prophylaxis after cardiovascular surgery and its effect on surgical site infections and antimicrobial resistance. Circulation 2000; 101: 2916-21.

11 Dellit TH, Chan JD, Fulton C, et al. Reduction in Clostridium difficile infections among neurosurgical patients associated with discontinuation of antimicrobial prophylaxis for the duration of external ventricular drain placement. Infect Control Hosp Epidemiol 2014; 35: 589-90.

12 WHO. Global guidelines for the prevention of surgical site infection. Geneva: World Health Organization, 2018.

13 Leaper D, Burman-Roy S, Palanca A, et al. Prevention and treatment of surgical site infection: summary of NICE guidance. BMJ 2008; 337: a1924.

14 Berríos-Torres SI, Umscheid CA, Bratzler DW, et al. Centers for Disease Control and Prevention guideline for the prevention of surgical site infection, 2017. JAMA Surg 2017; 152: 784-91.
15 Fadare JO, Ogunleye O, Iliyasu G, et al. Status of antimicrobial stewardship programmes in Nigerian tertiary healthcare facilities: findings and implications. J Glob Antimicrob Resist 2019; 17: 132-36.

16 Kalungia AC, Mwambula H, Munkombwe D, et al. Antimicrobial stewardship knowledge and perception among physicians and pharmacists at leading tertiary teaching hospitals in Zambia: implications for future policy and practice. J Chemother 2019; 31: $378-87$.

17 WHO. Web appendix 25. Summary of a systematic review on surgical antibiotic prophylaxis prolongation. In global guidelines for the prevention of surgical site infection. Geneva: World Health Organization, 2018. https://www.ncbi.nlm.nih.gov/books/ NBK536429/ (accessed April 28, 2010).

18 de Santibañes M, Glinka J, Pelegrini P, et al. Extended antibiotic therapy versus placebo after laparoscopic cholecystectomy for mild and moderate acute calculous cholecystitis: a randomized doubleblind clinical trial. Surgery 2018; 164: 24-30.

19 Sugawara G, Yokoyama Y, Ebata T, et al. Duration of antimicrobial prophylaxis in patients undergoing major hepatectomy with extrahepatic bile duct resection: a randomized controlled trial. Ann Surg 2018; 267: 142-48.

20 Moher D, Liberati A, Tetzlaff J, Altman DG. Preferred reporting items for systematic reviews and meta-analyses: the PRISMA statement. BMJ 2009; 339: b2535.

21 Higgins J, Green S. Cochrane handbook for systematic reviews of interventions. Hoboken, NJ: Wiley-Blackwell, 2011.

22 Mangram AJ, Horan TC, Pearson ML, Silver LC, Jarvis WR. Guideline for prevention of surgical site infection, 1999. Centers for Disease Control and Prevention (CDC) Hospital Infection Control Practices Advisory Committee. Am J Infect Control 1999; 27: 97-134.

23 Jackson JL, Kuriyama A, Anton A, et al. The accuracy of Google Translate for abstracting data from non-English-language trials for systematic reviews. Ann Intern Med 2019; 171: 677.

24 Wishart DS, Knox C, Guo AC, et al. DrugBank: a knowledgebase for drugs, drug actions and drug targets. Nucleic Acids Res 2008; 36 (suppl 1): D901-06.

25 Peters JL, Sutton AJ, Jones DR, Abrams KR, Rushton L. Contour-enhanced meta-analysis funnel plots help distinguish publication bias from other causes of asymmetry. J Clin Epidemiol 2008; 61: 991-96.

26 DerSimonian R, Laird N. Meta-analysis in clinical trials. Control Clin Trials 1986; 7: 177-88.

27 Turner RM, Davey J, Clarke MJ, Thompson SG, Higgins JP. Predicting the extent of heterogeneity in meta-analysis, using empirical data from the Cochrane Database of Systematic Reviews. Int J Epidemiol 2012; 41: 818-27

28 Thompson SG, Higgins JP. How should meta-regression analyses be undertaken and interpreted? Stat Med 2002; 21: 1559-73.

29 Borenstein M, Hedges LV, Higgins JPT, Rothstein HR. introduction to meta-analysis. Chichester: John Wiley and Sons, 2009.

30 Schünemann H, Brożek J, Guyatt G, Oxman A. GRADE handbook October 2013. https://gdt.gradepro.org/app/handbook/handbook. html (accessed April 28, 2020).

31 Organisation for Economic Co-operation and Development Stemming the superbug tide: just a few dollars more. Nov 7, 2018. https://www.oecd.org/health/stemming-the-superbug-tide9789264307599-en.htm (accessed April 28, 2020).

32 Cunha CB. The pharmacoeconomic aspects of antibiotic stewardship programs. Med Clin North Am 2018; 102: 937-46.

33 Dubberke ER, Olsen MA. Burden of Clostridium difficile on the healthcare system. Clin Infect Dis 2012; 55 (suppl 2): S88-92.

34 Fishman N. Policy statement on antimicrobial stewardship by the Society for Healthcare Epidemiology of America (SHEA), the Infectious Diseases Society of America (IDSA), and the Pediatric Infectious Diseases Society (PIDS). Infect Control Hosp Epidemiol 2012; 33: 322-27.

35 Nelson RL, Gladman E, Barbateskovic M. Antimicrobial prophylaxis for colorectal surgery. Cochrane Database Syst Rev 2014; 5: CD001181.

36 Naimi-Akbar A, Hultin M, Klinge A, Klinge B, Tranæus S, Lund B. Antibiotic prophylaxis in orthognathic surgery: a complex systematic review. PLoS One 2018; 13: e0191161.

37 Burke JF. The effective period of preventive antibiotic action in experimental incisions and dermal lesions. Surgery 1961; 50: 161-68. 
Articles

38 Bratzler DW, Houck PM, Richards C, et al. Use of antimicrobial prophylaxis for major surgery: baseline results from the National Surgical Infection Prevention Project. Arch Surg 2005; 140: 174-82.

39 Stewart LA, Tierney JF. To IPD or not to IPD? Advantages and disadvantages of systematic reviews using individual patient data. Eval Health Prof 2002; 25: 76-97.

40 Knobloch K, Gohritz A, Vogt PM. CONSORT and QUOROM statements revisited: standards of reporting of randomized controlled trials in general surgery. Ann Surg 2008; 248: 1106-07.
41 Moher D, Hopewell S, Schulz KF, et al. CONSORT 2010 explanation and elaboration: updated guidelines for reporting parallel group randomised trials. BMJ 2010; 340: c869.

42 Bruce J, Russell EM, Mollison J, Krukowski ZH. The measurement and monitoring of surgical adverse events. Health Technol Assess 2001; 5: 1-194. 